PROCEEDINGS OF THE

AMERICAN MATHEMATICAL SOCIETY

Volume 124, Number 7, July 1996

\title{
MULTIPLE PATH-VALUED CONDITIONAL YEH-WIENER INTEGRALS
}

\author{
CHULL PARK AND DAVID SKOUG
}

(Communicated by J. Marshall Ash)

\begin{abstract}
In this paper we establish various results involving parallel linevalued conditional Yeh-Wiener integrals of the type $E\left(F(x) \mid x\left(s_{j}, \cdot\right)=\eta_{j}(\cdot)\right.$, $j=1, \ldots, n)$ where $0<s_{1}<\cdots<s_{n}$. We then develop a formula for converting these multiple path-valued conditional Yeh-Wiener integrals into ordinary Yeh-Wiener integrals. Next, conditional Yeh-Wiener integrals for functionals $F$ of the form

$$
F(x)=\exp \left\{\int_{0}^{S} \int_{0}^{T} \phi(s, t, x(s, t)) d t d s\right\}
$$

are evaluated by solving an appropriate Wiener integral equation. Finally, a Cameron-Martin translation theorem is obtained for these multiple pathvalued conditional Yeh-Wiener integrals.
\end{abstract}

\section{INTRODUCTION}

For $Q=[0, S] \times[0, T]$ let $C(Q)$ denote Yeh-Wiener space, i.e., the space of all real-valued continuous functions $x(s, t)$ on $Q$ such that $x(0, t)=x(s, 0)=0$ for every $(s, t)$ in $Q$. Yeh [11] defined a Gaussian measure $m_{y}$ on $C(Q)$ (later modified in [14]) such that as a stochastic process $\{x(s, t),(s, t) \in Q\}$ has mean $E[x(s, t)]=$ $\int_{C(Q)} x(s, t) m_{y}(d x)=0$ and covariance $E[x(s, t) x(u, v)]=\min \{s, u\} \min \{t, v\}$. Let $C_{W} \equiv C[0, T]$ denote the standard Wiener space on $[0, T]$ with Wiener measure $m_{w}$. Yeh [13] introduced the concept of the conditional Wiener integral of $F$ given $X$, $E(F \mid X)$, and for the case $X(x)=x(T)$ obtained some very useful results including a Kac-Feynman integral equation.

A very important class of functions in quantum mechanics consists of functions on $C[0, T]$ of the type

$$
G(x)=\exp \left\{\int_{0}^{T} \theta(s, x(s)) d s\right\}
$$

where $\theta:[0, T] \times \mathbb{R} \rightarrow \mathbb{C}$.

Received by the editors December 14, 1994.

1991 Mathematics Subject Classification. Primary 28C20, $60 J 65$.

Key words and phrases. Yeh-Wiener integral, conditional Yeh-Wiener integral, Wiener integral equation, Cameron-Martin translation theorem. 
Yeh [13] shows that under suitable regularity conditions on $\theta$, the conditional Wiener integral

$$
H(t, \xi)=(2 \pi t)^{-1 / 2} \exp \left\{-\frac{\xi^{2}}{2 t}\right\} E\left(\exp \left\{\int_{0}^{t} \theta(s, x(s)) d s\right\} \mid x(t)=\xi\right)
$$

satisfied the Kac-Feynman integral equation

$$
\begin{aligned}
H(t, \xi)= & (2 \pi t)^{-1 / 2} \exp \left\{-\frac{\xi^{2}}{2 t}\right\} \\
& +\int_{0}^{t}[2 \pi(t-s)]^{-1 / 2} \int_{\mathbb{R}} \theta(s, \eta) H(s, \eta) \exp \left\{-\frac{(\eta-\xi)^{2}}{2(t-s)}\right\} d \eta d s
\end{aligned}
$$

whose solution can be expressed as an infinite series of terms involving Lebesgue integrals. Then using (1.1), one can use the series solution of (1.2) to evaluate the conditional Wiener integral

$$
E\left(\exp \left\{\int_{0}^{t} \theta(s, x(s)) d s\right\} \mid x(t)=\xi\right) .
$$

The corresponding problem in Yeh-Wiener space, namely to evaluate

$$
E\left(\exp \left\{\int_{0}^{t} \int_{0}^{s} \phi(u, v, x(u, v)) d u d v\right\} \mid x(x, t)=\xi\right),
$$

turned out to be substantially different from the corresponding one-parameter problem. After many attempts to solve this problem by several mathematicians, the first really successful solution was given by Park and Skoug [9] by introducing a sample path-valued conditional Yeh-Wiener integral of the type

$$
E\left(\exp \left\{\int_{0}^{t} \int_{0}^{s} \phi(u, v, x(u, v)) d u d v\right\} \mid x(s, \cdot)=\eta(\cdot)\right),
$$

which satisfies a Wiener integral equation similar to that of Cameron and Storvick [2]. The Wiener integral equation is then solved to evaluate (1.4), and finally (1.3) is obtained by integrating (1.4) appropriately.

In this paper we consider parallel line-valued conditional Yeh-Wiener integrals of the type

$$
E\left(F(x) \mid x\left(s_{j}, \cdot\right)=\eta_{j}(\cdot), j=1, \ldots, n\right),
$$

where $F \in L_{1}\left(C(Q), m_{y}\right)$ and $0=s_{0}<s_{1}<\cdots<s_{n}=S$. In section 2 we develop a formula for converting these conditional Yeh-Wiener integrals into ordinary YehWiener integrals and in section 3 we use this formula to evaluate (1.5) for various functionals $F$. In section 4, we evaluate (1.5) for functionals $F$ of the form $F(x)=$ $\exp \left\{\int_{0}^{S} \int_{0}^{T} \phi(u, t, x(u, t)) d t d u\right\}$ by solving an appropriate Wiener integral equation. Finally, in section 5, a Cameron-Martin type translation theorem is obtained for conditional Yeh-Wiener integrals of the type (1.5).

\section{Parallel Line-Valued CONditional Yeh-Wiener integrals}

Let $\sigma: 0=s_{0}<s_{1}<\cdots<s_{n}=S$ be any partition of $[0, S]$. For $x \in C(Q)$, define $X_{\sigma}(x)$ by

$$
X_{\sigma}(x)=\left(x\left(s_{1}, \cdot\right), \ldots, x\left(s_{n}, \cdot\right)\right) .
$$


We also define an $s$-sectional function $x_{\sigma}$ of $x \in C(Q)$ by

$$
\begin{aligned}
& x_{\sigma}(s, \cdot)=x\left(s_{j-1}, \cdot\right)+\frac{s-s_{j-1}}{s_{j}-s_{j-1}}\left[x\left(s_{j}, \cdot\right)-x\left(s_{j-1}, \cdot\right)\right], \\
& s_{j-1} \leq s \leq s_{j}, j=1, \ldots, n .
\end{aligned}
$$

Similarly for $\bar{\eta}(\cdot)=\left(\eta_{1}(\cdot), \ldots, \eta_{n}(\cdot)\right), \eta_{j}(\cdot) \in C[0, T]$, we define

$$
\begin{aligned}
& \bar{\eta}_{\sigma}(s, \cdot)=\eta_{j-1}(\cdot)+\frac{s-s_{j-1}}{s_{j}-s_{j-1}}\left[\eta_{j}(\cdot)-\eta_{j-1}(\cdot)\right], \\
& \qquad s_{j-1} \leq s \leq s_{j}, j=1, \ldots, n,
\end{aligned}
$$

where $\eta_{0}(\cdot) \equiv 0$.

Our first result, which plays a key role throughout this paper, involves the stochastic independence between $x-x_{\sigma}$ and $X_{\sigma}(x)$ and between $x-x_{\sigma}$ and $x$.

Theorem 1. If $\{x(s, t),(s, t) \in Q\}$ is the standard Yeh-Wiener process, then $x-x_{\sigma}$ and $X_{\sigma}(x)$ are stochastically independent on $Q$. In addition $x-x_{\sigma}$ and $x$ are stochastically independent on distinct rectangles $\left[s_{i-1}, s_{i}\right] \times[0, T]$ and $\left[s_{j-1}, s_{j}\right] \times$ $[0, T]$ of $Q$, where the $s_{k}$ are partition points in $\sigma$.

Proof. For $s_{j-1} \leq s \leq s_{j}$ we note that

$$
x(s, t)-x_{\sigma}(s, t)=x(s, t)-x\left(s_{j-1}, t\right)-\frac{s-s_{j-1}}{s_{j}-s_{j-1}}\left[x\left(s_{j}, t\right)-x\left(s_{j-1}, t\right)\right] .
$$

Thus, to show that $x-x_{\sigma}$ and $X_{\sigma}(x)$ are independent, it is sufficient to show that (2.4) is independent of $x\left(s_{k}, t^{\prime}\right)$ for $k=1, \ldots, n$. Using the formula

$$
E[x(s, t) x(u, v)]=\min \{s, u\} \min \{t, v\}
$$

it is easy to establish that

$$
E\left\{x\left(s_{k}, t^{\prime}\right)\left[x(s, t)-x_{\sigma}(s, t)\right]\right\}=0 .
$$

Since uncorrelated Gaussian processes are independent, it follows that $x-x_{\sigma}$ and $X_{\sigma}(x)$ are independent. The independence of $x-x_{\sigma}$ and $x$ on distinct rectangles of $Q$ follows similarly.

The following corollary is an immediate consequence of Theorem 1.

Corollary. The two processes $x-x_{\sigma}$ and $x_{\sigma}$ are independent Gaussian processes on $Q$.

Our next theorem, in which we express a conditional Yeh-Wiener integral in terms of a Yeh-Wiener integral, also plays a key role throughout this paper.

Theorem 2. Let $F \in L_{1}(C(Q))$. Then

$$
E\left(F(x) \mid X_{\sigma}(x)=\bar{\eta}(\cdot)\right)=E\left[F\left(x-x_{\sigma}+\bar{\eta}_{\sigma}\right] .\right.
$$

Proof. Under the conditioning $X_{\sigma}(x)=\bar{\eta}(\cdot)$, we have that $x_{\sigma}=\bar{\eta}_{\sigma}$, and so

$$
E\left(F(x) \mid X_{\sigma}(x)=\bar{\eta}(\cdot)\right)=E\left(f\left(x-x_{\sigma}+\bar{\eta}_{\sigma}\right) \mid X_{\sigma}(x)=\bar{\eta}(\cdot)\right) .
$$

The result now follows because $x-x_{\sigma}$ and $X_{\sigma}(x)$ are independent by Theorem 1.

The following theorem shows how to recover $E[F(x)]$ from the conditional YehWiener integral. 
Theorem 3. Let $F \in L_{1}(C(Q))$ and let $w_{1}, \ldots, w_{n}$ be $n$ independent Wiener processes on $[0, T]$ which are independent of the Yeh-Wiener process $x$. Let $\bar{\eta}(\cdot)=$ $\left(\eta_{1}(\cdot), \ldots, \eta_{n}(\cdot)\right)$ where, for $j=1, \ldots, n, \eta_{j}(\cdot)=\sum_{k=1}^{j}\left(s_{k}-s_{k-1}\right)^{1 / 2} w_{k}(\cdot)$. Then

$$
E_{w_{1}, \ldots, w_{n}}\left\{E\left(F(x) \mid X_{\sigma}(x)=\bar{\eta}(\cdot)\right)\right\}=E[F(x)] .
$$

Proof. By Theorem 2, we have that $E\left(F(x) \mid X_{\sigma}(x)=\bar{\eta}(\cdot)\right)=E\left[F\left(x-x_{\sigma}+\bar{\eta}_{\sigma}\right)\right]$. Let $y(s, t)=x(s, t)-x_{\sigma}(s, t)+\bar{\eta}_{\sigma}(s, t)$. Then $y$, as a process depending on $x$ and the $w_{j}$ 's, has mean $E[y(s, t)]=0$ and covariance $E[y(s, t) y(u, v)]=\min \{s, u\} \min \{t, v\}$. Thus $y$ is a standard Yeh-Wiener process on $Q$, and so

$$
E_{w_{1}, \ldots, w_{n}}\left\{E_{x}\left[F\left(x-x_{\sigma}+\bar{\eta}_{\sigma}\right)\right]\right\}=\int_{C(Q)} F(y) m_{y}(d y)=E[F(x)],
$$

which completes the proof.

In the following sections, we shall consider stochastic integrals of $h \in L_{2}(Q)$ with respect to $x_{\sigma}$, defined by $(2.2)$. Such stochastic integrals have convenient representations with respect to $\hat{h}$, the $s$-sectional average of $h$.

Definition. Let $\sigma$ be as before. For each function $h \in L_{2}(Q)$, define the $s$-sectional average of $h$ by

$$
\hat{h}(s, t)= \begin{cases}\frac{1}{s_{j}-s_{j-1}} \int_{s_{j-1}}^{s_{j}} h(u, t) d u, & s_{j-1}<s \leq s_{j}, j=1,2, \ldots, n, \\ 0, & s t=0 .\end{cases}
$$

The following theorem gives some useful and interesting formulas involving $\hat{h}, h$ and $x_{\sigma}$. The proof is rather straightforward and hence omitted. A similar observation was made in $[8$, p. 456]. In particular, the observation in (2.9) that the stochastic integrals $\int_{q} h d x_{\sigma}$ and $\int_{q} \hat{h} d x_{\sigma}$ both equal the stochastic integral $\int_{q} \hat{h} d x$ is very useful in evaluating various expectations.

Theorem 4. Let $h \in L_{2}(Q)$. Then

$$
\begin{gathered}
\int_{Q} h(s, t) \hat{h}(s, t) d s d t=\int_{Q}[\hat{h}(s, t)]^{2} d s d t \\
\|h\|_{1} \geq\|\hat{h}\|_{1} \text { and }\|h\|_{1}=\|\hat{h}\|_{1} \text { if either } h \geq 0 \text { or } h \leq 0 \text { a.e. on } Q, \\
\|h-\hat{h}\|_{2}^{2}=\|h\|_{2}^{2}-\|\hat{h}\|_{2}^{2} \geq 0
\end{gathered}
$$

and

$$
\int_{Q} h d x_{\sigma}=\int_{Q} \hat{h} d x=\int_{Q} \hat{h} d x_{\sigma} \quad \text { for every } x \in C(Q)
$$

\section{EXAMPLES}

In this section we illustrate that Theorem 2 makes the evaluation of the multiple path-valued conditional Yeh-Wiener integral of certain functionals rather simple.

Example 1. For $x \in C(Q)$, let $F(x)=\int_{Q} x(s, t) d s d t$. Then, by Theorem 2 and the Fubini Theorem, we obtain

$$
I \equiv E\left(\int_{Q} x(s, t) d s d t \mid X_{\sigma}(x)=\bar{\eta}(\cdot)\right)=\sum_{j=1}^{n} \frac{s_{j}-s_{j-1}}{2} \int_{0}^{T}\left[\eta_{j}(t)-\eta_{j-1}(t)\right] d t .
$$


In particular, if $n=1$, then $I=\frac{S}{2} \int_{0}^{T} \eta_{1}(t) d t$, which agrees with the computation in $[9]$.

Example 2. Let $F(x)=\int_{Q} x^{2}(s, t) d s d t$. Then, proceeding as in Example 1 above, we obtain

$$
\begin{aligned}
I & \equiv E\left(\int_{Q} x^{2}(s, t) d s d t \mid X_{\sigma}(x)=\bar{\eta}(\cdot)\right) \\
& =\frac{T^{2}}{12} \sum_{j=1}^{n}\left(s_{j}-s_{j-1}\right)^{2}+\int_{Q} \bar{\eta}_{\sigma}^{2}(s, t) d s d t .
\end{aligned}
$$

If $n=1$, then $I=\frac{S^{2} T^{2}}{12}+\frac{S}{3} \int_{0}^{T} \eta_{1}^{2}(t) d t$, which agrees with the calculation in [9].

Example 3. Let $M\left(L_{2}(Q)\right)$ be the class of all countably additive complex-valued Borel measures on $L_{2}(Q)$. The Banach algebra $\mathcal{S}(2)$ consists of the functionals on $C(Q)$ of the form

$$
F(x)=\int_{L_{2}(Q)} \exp \left\{i \int_{Q} v(s, t) d x(s, t)\right\} d \delta(v)
$$

with $\delta \in M\left(L_{2}(Q)\right)$. Using Theorem 2, the Fubini Theorem, and letting $\hat{v}$ denote the $s$-sectional average function of $v$ given by $(2.5)$, we see that for each $F \in \mathcal{S}(2)$,

$$
\begin{aligned}
J & \equiv E\left(F(x) \mid X_{\sigma}(x)=\bar{\eta}(\cdot)\right) \\
& =\int_{L_{2}(Q)} \exp \left\{i \int_{Q} v d \bar{\eta}_{\sigma}\right\} E\left[\exp \left\{i \int_{Q}(v-\hat{v}) d x\right\}\right] d \delta(v) .
\end{aligned}
$$

Hence, an application of a familiar Yeh-Wiener integration formula yields

$$
J=\int_{L_{2}(Q)} \exp \left\{-\frac{1}{2}\left(\|v\|^{2}-\|\hat{v}\|^{2}\right)\right\} \exp \left\{i \int_{Q} v d \bar{\eta}_{\sigma}\right\} d \delta(v)
$$

Example 4. In this example we show that Theorem 3 holds for all $F \in \mathcal{S}(2)$ in Example 3 above. For let

$$
\eta_{j}(\cdot)=\sum_{k=1}^{j}\left(s_{k}-s_{k-1}\right)^{1 / 2} w_{k}(\cdot), \quad j=1, \ldots, n
$$

Then, using (2.3) we have,

$$
\int_{Q} v(s, t) d \bar{\eta}_{\sigma}(s, t)=\sum_{j=1}^{n} \int_{0}^{T}\left[\int_{s_{j-1}}^{s_{j}} v(s, t)\left(s_{j}-s_{j-1}\right)^{-1 / 2} d s\right] d w_{j}(t) .
$$


Substituting this into (3.1), and then integrating with respect to $w_{1}, \ldots, w_{n}$, yields

$$
\begin{aligned}
E_{w_{1}, \ldots, w_{n}}(J)= & \int_{L_{2}(Q)} \exp \left\{-\frac{1}{2}\left[\|v\|_{2}^{2}-\|\hat{v}\|_{2}^{2}\right]\right\} \\
& \cdot \exp \left\{-\frac{1}{2} \sum_{j=1}^{n} \int_{0}^{T}\left[\int_{s_{j-1}}^{s_{j}} v(s, t)\left(s_{j}-s_{j-1}\right)^{-1 / 2} d s\right]^{2} d t\right\} d \delta(v) \\
= & \int_{L_{2}(Q)} \exp \left\{-\frac{1}{2}\left[\|v\|_{2}^{2}-\|\hat{v}\|_{2}^{2}\right]\right\} \exp \left\{-\frac{1}{2} \int_{Q} \hat{v}^{2}(s, t) d s d t\right\} d \delta(v) \\
= & \int_{L_{2}(Q)} \exp \left\{-\frac{1}{2}\|v\|_{2}^{2}\right\} d \delta(v) \\
= & E\left[\int_{L_{2}(Q)} \exp \left\{i \int_{Q} v(s, t) d x(s, t)\right\} d \delta(v)\right]=E[F(x)] .
\end{aligned}
$$

4. Evaluation of $E\left(\exp \left\{\int_{Q} \phi(u, t, x(u, t)) d u d t\right\} \mid X_{\sigma}(x)=\bar{\eta}(\cdot)\right)$

Let $\phi(s, t, v)$ be a bounded continuous function on $Q \times \mathbb{R}$, and let

$$
\theta(u, x(u, \cdot))=\int_{0}^{T} \phi(u, t, x(u, t)) d t .
$$

Park and Skoug [9] have shown that the function $G$ defined on $[0, S] \times C[0, T]$ by

$$
G(s, \eta(\cdot)) \equiv E\left(\exp \left\{\int_{0}^{s} \theta(u, x(u, \cdot)) d u\right\} \mid x(s, \cdot)=\eta(\cdot)\right)
$$

satisfies the Wiener integral equation

$$
\begin{aligned}
G(s, \eta(\cdot))=1+\int_{0}^{s} E_{w}[\theta & \left(u, \sqrt{u\left(1-\frac{u}{s}\right)} w(\cdot)+\frac{u}{s} \eta(\cdot)\right) \\
\cdot & \left.G\left(u, \sqrt{u\left(1-\frac{u}{s}\right)} w(\cdot)+\frac{u}{s} \eta(\cdot)\right)\right] d u
\end{aligned}
$$

whose solution is given by

$$
G(s, \eta(\cdot))=\sum_{k=1}^{\infty} H_{k}(s, \eta(\cdot))
$$

where the sequence $\left\{H_{k}\right\}$ is given inductively by $H_{0}(s, \eta(\cdot)) \equiv 1$, and

$$
H_{k+1}(s, \eta(\cdot))=\int_{0}^{s} E_{w}\left[\left(\theta \cdot H_{k}\right)\left(u, \sqrt{u\left(1-\frac{u}{s}\right)} w(\cdot)+\frac{u}{s} \eta(\cdot)\right)\right] d u,
$$

where $\left(\theta \cdot H_{k}\right)(u, v)=\theta(u, v) H_{k}(u, v)$. Furthermore, if $|\theta(s, v)| \leq M$ on $[0, S] \times \mathbb{R}$, 
then

$$
\left|H_{k}(s, \eta(\cdot))\right| \leq \frac{(M s)^{k}}{k !} \leq \frac{(M S)^{k}}{k !} \text { for } k=0,1,2, \ldots
$$

Under the same assumptions on $\phi$ and $\theta$, we now proceed to find the multiconditional expectation

$$
E\left(\exp \left\{\int_{0}^{S} \theta(u, x(u, \cdot)) d u\right\} \mid X_{\sigma}(x)=\bar{\eta}(\cdot)\right)
$$

We start with the following lemmas.

Lemma 1. Let $\sigma: 0=s_{0}<s_{1}<\cdots<s_{n}=S$ be any partition on $[0, S]$, and let $\theta$ be given by (4.1). Then for $j=1,2, \ldots, n$,

$$
\begin{aligned}
& E\left(\exp \left\{\int_{s_{j-1}}^{s_{j}} \theta(u, x(u, \cdot)) d u\right\} \mid X_{\sigma}(x)=\bar{\eta}(\cdot)\right) \\
& \quad=E\left(\exp \left\{\int_{s_{j-1}}^{s_{j}} \theta(u, x(u, \cdot)) d u\right\} \mid x\left(s_{j-1}, \cdot\right)=\eta_{j-1}(\cdot), x\left(s_{j}, \cdot\right)=\eta_{j}(\cdot)\right)
\end{aligned}
$$

Proof. By Theorem 2, (2.2), and (2.3),

$$
\begin{aligned}
& E\left(\exp \left\{\int_{s_{j-1}}^{s_{j}} \theta(u, x(u, \cdot)) d u\right\} \mid X_{\sigma}(x)=\bar{\eta}(\cdot)\right) \\
& =E\left[\operatorname { e x p } \left\{\int _ { s _ { j - 1 } } ^ { s _ { j } } \theta \left(u, x(u, \cdot)-x\left(s_{j-1}, \cdot\right)-\frac{u-s_{j-1}}{s_{j}-s_{j-1}}\left[x\left(s_{j}, \cdot\right)-x\left(s_{j-1}, \cdot\right)\right]\right.\right.\right. \\
& \left.\left.\left.\quad+\eta_{j-1}(\cdot)+\frac{u-s_{j-1}}{s_{j}-s_{j-1}}\left[\eta_{j}(\cdot)-\eta_{j-1}(\cdot)\right]\right) d u\right\}\right] \\
& =E\left(\exp \left\{\int_{s_{j-1}}^{s_{j}} \theta(u, x(u, \cdot)) d u\right\} \mid x\left(s_{j-1}, \cdot\right)=\eta_{j-1}(\cdot), x\left(s_{j}, \cdot\right)=\eta_{j}(\cdot)\right) .
\end{aligned}
$$

Lemma 2. Under the assumptions of Lemma 1,

$$
\begin{aligned}
E\left(\exp \left\{\int_{0}^{S} \theta(u, x(u, \cdot)) d u\right\} \mid X_{\sigma}(x)=\bar{\eta}(\cdot)\right) \\
=\prod_{j=1}^{n} E\left(\exp \left\{\int_{s_{j-1}}^{s_{j}} \theta(u, x(u, \cdot)) d u\right\} \mid x\left(s_{j-1}, \cdot\right)=\eta_{j-1}(\cdot), x\left(s_{j}, \cdot\right)=\eta_{j}(\cdot)\right) .
\end{aligned}
$$


Proof. One can easily establish that the processes $\left\{x(s, \cdot)-x_{\sigma}(s, \cdot), s_{j-1} \leq s \leq s_{j}\right\}$, $j=1, \ldots, n$, are independent. Thus

$$
\begin{gathered}
E\left(\exp \left\{\int_{0}^{S} \theta(u, x(u, \cdot)) d u\right\} \mid X_{\sigma}(x)=\bar{\eta}(\cdot)\right) \\
=E\left[\prod_{j=1}^{n} \exp \left\{\int_{s_{j-1}}^{s_{j}} \theta\left(u, x(u, \cdot)-x_{\sigma}(u, \cdot)+\bar{\eta}_{\sigma}(u, \cdot)\right) d u\right\}\right] \\
=\prod_{j=1}^{n} E\left(\exp \left\{\int_{s_{j-1}}^{s_{j}} \theta(u, x(u, \cdot)) d u\right\} \mid X_{\sigma}(x)=\bar{\eta}(\cdot)\right) .
\end{gathered}
$$

The result now follows by Lemma 1 .

Next, consider the conditional expectation for $s_{1}<s \leq s_{2}$,

$$
I \equiv E\left(\exp \left\{\int_{s_{1}}^{s} \theta(u, x(u, \cdot)) d u\right\} \mid x\left(s_{1}, \cdot\right)=\eta_{1}(\cdot), x(s, \cdot)=\eta(\cdot)\right) .
$$

Since the Yeh-Wiener process has stationary increments in each components, it follows that

$$
\begin{aligned}
I & =E\left(\exp \left\{\int_{0}^{s-s_{1}} \theta\left(u+s_{1}, x(u, \cdot)+\eta_{1}(\cdot)\right) d u\right\} \mid x\left(s-s_{1}, \cdot\right)=\eta(\cdot)-\eta_{1}(\cdot)\right) \\
& \equiv G^{*}\left(s-s_{1}, \eta(\cdot)-\eta_{1}(\cdot) ; s_{1}, \eta_{1}(\cdot)\right) .
\end{aligned}
$$

Comparing (4.7) and (4.2), one can easily deduce that the function $G^{*}$ satisfies the Wiener integral equation

$$
\begin{gathered}
G^{*}\left(s-s_{1}, \eta(\cdot)-\eta_{1}(\cdot) ; s_{1}, \eta_{1}(\cdot)\right) \\
=1+\int_{0}^{s-s_{1}} E_{w}\left[\theta\left(u+s_{1}, \sqrt{u\left(1-\frac{u}{s-s_{1}}\right)} w(\cdot)+\frac{u}{s-s_{1}}\left[\eta(\cdot)-\eta_{1}(\cdot)\right]+\eta_{1}(\cdot)\right)\right. \\
\left.\cdot G^{*}\left(u, \sqrt{u\left(1-\frac{u}{s-s_{1}}\right)} w(\cdot)+\frac{u}{s-s_{1}}\left[\eta(\cdot)-\eta_{1}(\cdot)\right] ; s_{1}, \eta_{1}(\cdot)\right)\right] d u
\end{gathered}
$$

whose solution is given by

$$
G^{*}\left(s-s_{1}, \eta(\cdot)-\eta_{1}(\cdot) ; s_{1}, \eta_{1}(\cdot)\right)=\sum_{k=0}^{\infty} H_{k}^{*}\left(s-s_{1}, \eta(\cdot)-\eta_{1}(\cdot) ; s_{1}, \eta_{1}(\cdot)\right)
$$

where the sequence $\left\{H_{k}^{*}\right\}$ is given inductively by $H_{0}^{*} \equiv 1$, and

$$
\begin{aligned}
& H_{k+1}^{*}\left(s-s_{1}, \eta(\cdot)-\eta_{1}(\cdot) ; s_{1}, \eta_{1}(\cdot)\right) \\
& =\int_{0}^{s-s_{1}} E_{w}\left[\theta\left(u+s_{1}, \sqrt{u\left(1-\frac{u}{s-s_{1}}\right)} w(\cdot)+\frac{u}{s-s_{1}}\left[\eta(\cdot)-\eta_{1}(\cdot)\right]+\eta_{1}(\cdot)\right)\right. \\
& \left.\cdot H_{k}^{*}\left(u, \sqrt{u\left(1-\frac{u}{s-s_{1}}\right)} w(\cdot)+\frac{u}{s-s_{1}}\left[\eta(\cdot)-\eta_{1}(\cdot)\right] ; s_{1}, \eta_{1}(\cdot)\right)\right] d u .
\end{aligned}
$$

Obviously each $H_{k}^{*}$ is bounded by the bound of $H_{k}$ given in formula (4.5). Thus, under the same assumptions on $\theta$, the series in formula (4.9) converges abso- 
lutely and uniformly for $s_{1} \leq s \leq s_{2}$. In particular, the series corresponding to $G^{*}\left(s_{2}-s_{1}, \eta_{2}(\cdot)-\eta_{1}(\cdot) ; s_{1}, \eta_{1}(\cdot)\right)$ converges absolutely.

Exactly the same argument gives rise to an absolutely convergent series expansion for

$$
\begin{aligned}
& G^{*}\left(s_{j}-s_{j-1}, \eta_{j}(\cdot)-\eta_{j-1}(\cdot) ; s_{j-1}, \eta_{j-1}(\cdot)\right) \\
& \quad=E\left(\exp \left\{\int_{s_{j-1}}^{s_{j}} \theta(u, x(u, \cdot)) d u\right\} \mid x\left(s_{j-1}, \cdot\right)=\eta_{j-1}(\cdot), x\left(s_{j}, \cdot\right)=\eta_{j}(\cdot)\right)
\end{aligned}
$$

for $j=1,2, \ldots, n$. Hence by Lemma 2 , we finally obtain that

$$
\begin{gathered}
E\left(\exp \left\{\int_{0}^{S} \int_{0}^{T} \phi(u, t, x(u, t)) d t d u\right\} \mid x_{\sigma}(x)=\bar{\eta}(\cdot)\right) \\
=E\left(\exp \left\{\int_{0}^{S} \theta(u, x(u, \cdot)) d u\right\} \mid X_{\sigma}(x)=\bar{\eta}(\cdot)\right) \\
=\prod_{j=1}^{n} G^{*}\left(s_{j}-s_{j-1}, \eta_{j}(\cdot)-\eta_{j-1}(\cdot) ; s_{j-1}, \eta_{j-1}(\cdot)\right) .
\end{gathered}
$$

\section{Translation of Parallel Line-VAlued CONDITIONAL YEH-WIENER INTEGRALS}

The Cameron-Martin Translation Theorem for Yeh-Wiener integrals [12] states that if $x_{0}(s, t)=\int_{0}^{t} \int_{0}^{s} h(u, v) d u d v$ on $Q$ for $h \in L^{2}(Q)$, and if $T_{1}$ is the transformation of $C(Q)$ into itself defined by

$$
T_{1}(x)=x+x_{0} \quad \text { for } x \in C(Q),
$$

then, for any Yeh-Wiener integrable function $F$ on $C(Q)$ and any Yeh-Wiener measurable set $\Gamma$,

$$
\int_{\Gamma} F(z) m_{y}(d z)=\int_{T_{1}^{-1}(\Gamma)} F\left(x+x_{0}\right) J\left(x_{0}, x\right) m_{y}(d x),
$$

where

$$
J\left(x_{0}, x\right)=\exp \left\{-\frac{1}{2} \int_{Q} h^{2}(u, v) d u d v\right\} \exp \left\{-\int_{Q} h(u, v) d x(u, v)\right\} .
$$

In particular, if $\Gamma=C(Q)$, then (5.1) becomes

$$
E[F(z)]=E\left[F\left(x+x_{0}\right) J\left(x_{0}, x\right)\right] .
$$

The following theorem is the conditional version of (5.2).

Theorem 5. Let $h \in L_{2}(Q)$ be given and let $x_{0}(s, t)=\int_{0}^{t} \int_{0}^{s} h(u, v) d u d v$ on $Q$. Let $F \in L_{1}\left(C(Q), m_{y}\right)$. Then for each partition $\sigma: 0=s_{0}<s_{1}<\cdots<s_{n}=S$ of $[0, S]$,

$$
\begin{aligned}
& E\left(F(z) \mid X_{\sigma}(z)=\bar{\eta}(\cdot)\right) \\
& \quad=\exp \left\{-\frac{1}{2}\|\hat{h}\|_{2}^{2}+\int_{Q} h d \bar{\eta}_{\sigma}\right\} E\left(F\left(x+x_{0}\right) J\left(x_{0}, x\right) \mid X_{\sigma}\left(x+x_{0}\right)=\bar{\eta}(\cdot)\right) .
\end{aligned}
$$


Proof. First, using Theorem 3, we see that

$$
E\left(F(z) \mid X_{\sigma}(z)=\bar{\eta}(\cdot)\right)=E\left[F\left(z-z_{\sigma}+\bar{\eta}_{\sigma}\right)\right] .
$$

Since $\left(x+x_{0}\right)_{\sigma}=x_{\sigma}+\left(x_{0}\right)_{\sigma}$, we may apply (5.2) to get that

$$
E\left[F\left(z-z_{\sigma}+\bar{\eta}_{\sigma}\right)\right]=E\left[F\left(x+x_{0}-x_{\sigma}-\left(x_{0}\right)_{\sigma}+\bar{\eta}_{\sigma}\right) J\left(x_{0}, x\right)\right] .
$$

Next, we rewrite $J\left(x_{0}, x\right)$ in the form,

$$
\begin{aligned}
J\left(x_{0}, x\right)= & \exp \left\{-\frac{1}{2}\|h\|_{2}^{2}\right\} \exp \left\{-\int_{Q} h d\left(x-x_{\sigma}+\bar{\eta}_{\sigma}-\left(x_{0}\right)_{\sigma}\right)\right\} \\
& \cdot \exp \left\{-\int_{Q} h d x_{\sigma}\right\} \exp \left\{\int_{Q} h d \bar{\eta}_{\sigma}\right\} \exp \left\{-\int_{Q} h d\left(x_{0}\right)_{\sigma}\right\} .
\end{aligned}
$$

But $x-x_{\sigma}$ and $x_{\sigma}$ are independent processes on $Q$ by the Corollary to Theorem 1 , and so it follows from (5.3) and (5.4) that

$$
\begin{aligned}
& E\left[F\left(z-z_{\sigma}+\bar{\eta}_{\sigma}\right)\right] \\
& \left.=\exp \left\{-\frac{1}{2}\|h\|_{2}^{2}+\int_{Q} h d \bar{\eta}_{\sigma}-\int_{Q} h d\left(x_{0}\right)\right)_{\sigma}\right\} E\left[\exp \left\{-\int_{Q} h d x_{\sigma}\right\}\right] \\
& \cdot E\left[F\left(x+x_{0}-x_{\sigma}-\left(x_{0}\right)_{\sigma}+\bar{\eta}_{\sigma}\right) \exp \left\{-\int_{Q} h d\left(x-x_{\sigma}+\bar{\eta}_{\sigma}-\left(x_{0}\right)_{\sigma}\right)\right\}\right] .
\end{aligned}
$$

Since $\int_{Q} h d x_{\sigma}=\int_{Q} \hat{h} d x$ by (2.9), $\int_{Q} h d x_{\sigma}$ is a normal random variable with variance $\|\hat{h}\|_{2}^{2}$, and so

$$
E\left[\exp \left\{-\int_{Q} h d x_{\sigma}\right\}\right]=\exp \left\{\frac{1}{2}\|\hat{h}\|_{2}^{2}\right\} .
$$

Next, using (2.9), (2.6), and the definition of $x_{0}$, we see that

$$
\int_{Q} h d\left(x_{0}\right)_{\sigma}=\int_{Q} \hat{h} d\left(x_{0}\right)=\int_{Q} \hat{h} h=\|\hat{h}\|_{2}^{2} .
$$

Now, using Theorem 2, we obtain that

$$
\begin{aligned}
& E\left(F\left(x+x_{0}\right) J\left(x_{0}, x\right) \mid X_{\sigma}\left(x+x_{0}\right)=\bar{\eta}(\cdot)\right) \\
&=\exp \left\{-\frac{1}{2}\|h\|_{2}^{2}\right\} E[ F\left(x+x_{0}-x_{\sigma}-\left(x_{0}\right)_{\sigma}+\bar{\eta}_{\sigma}\right) \\
&\left.\cdot \exp \left\{-\int_{Q} h d\left(x-x_{\sigma}+\bar{\eta}_{\sigma}-\left(x_{0}\right)_{\sigma}\right)\right\}\right] .
\end{aligned}
$$

Finally, substitution of (5.6) through (5.8) into (5.5) yields

$$
\begin{aligned}
E\left[F\left(z-z_{\sigma}+\bar{\eta}_{\sigma}\right)\right]= & \exp \left\{-\frac{1}{2}\|\hat{h}\|_{2}^{2}+\int_{Q} h d \bar{\eta}_{\sigma}\right\} \\
& \cdot E\left(F\left(x+x_{0}\right) J\left(x_{0}, x\right) \mid X_{\sigma}\left(x+x_{0}\right)=\bar{\eta}(\cdot)\right),
\end{aligned}
$$

which completes the proof. 


\section{REFERENCES}

1. R. H. Cameron and D. A. Storvick, Two related integrals over spaces of continuous functions, Pacific J. Math. 55 (1974), 19-37. MR 51:5881

2. _ An operator-valued Yeh-Feynman integral and a Feynman integral equation, Japan J. Math. 6 (1980), 283-342. MR 84m:81065

3. K. S. Chang, J. M. Ahn, and J. S. Chang, An evaluation of the conditional Yeh-Wiener integral, Pacific J. Math. 124 (1986), 107-117. MR 88e:60094

4. D. M. Chung and J. M. Ahn, Conditional Yeh-Wiener integrals, J. Korean Math. Soc. 20 (1983), 209-221. MR 85j:28013

5. J. L. Doob, Stochastic processes, Wiley, New York, 1965 (originally published in 1953). MR 15: $445 \mathrm{~b}$

6. T. J. Huffman, C. Park and D. L. Skoug, Analytic Fourier-Feynman transforms and convolution, Trans. Amer. Math. Soc. 347 (1995), 661-673. MR 95d:28017

7. C. Park, On Fredholm transformations in Yeh-Wiener space, Pacific J. Math. 40 (1972), 173-195. MR 46:3739

8. C. Park and D. L. Skoug, Conditional Yeh-Wiemer integrals with vector valued conditioning functions, Proc. Amer. Math. Soc. 105 (1989), 450-461. MR 89m:60198

9. $ـ$ Sample path-valued conditional Yeh-Wiener integrals and a Wiener integral equation, Proc. Amer. Math. Soc. 115 (1992), 479-487. MR 92i:28016

10. _ An operator-valued Yeh-Wiener integral, and a Kac-Feynman Wiener-Integral equation, Proc. Amer. Math. Soc. 120 (1994), 929-942. MR 94h:28009

11. J. Yeh, Wiener measure in a space of functions of two variables, Trans. Amer. Math. Soc. 95 (1960), 433-450. MR 23:A2735

12. Cameron-Martin translation theorems in the Wiener space of functions of two variables, Trans. Amer. Math. Soc. 107 (1963), 409-420. MR 32:6565

13. Inversion of conditional Wiener integrals, Pacific J. Math. 59 (1975), 623-638. MR 52:10988

14. _ Stochastic processes and the Wiener integral, Marcel Dekker, New York, 1983.

Department of Mathematics \& Statistics, Miami University, Oxford, Ohio 45056

E-mail address: cpark@miavxl.acs.muohio.edu

Department of Mathematics \& Statistics, University of Nebraska, Lincoln, Nebraska 68588-0323

E-mail address: dskoug@unl.edu 